\title{
The ground is dominant in infants' perception of relative distance
}

\author{
Michael Kavšek • Carl E. Granrud
}

Published online: 10 November 2012

(C) Psychonomic Society, Inc. 2012

\begin{abstract}
When making relative distance judgments, adults attend to information provided by the ground surface and generally ignore information provided by ceiling surfaces. In the present study, we asked whether this ground dominance effect is present in infancy. Groups of 5- and 7-month-old infants viewed a display depicting textured ground and ceiling surfaces. Two toys, which were attached to vertical rods, were affixed to the display. The toys/rods were positioned so that one toy was specified as being nearer by the ground surface but farther away by the ceiling surface, while the other toy was specified as being farther away by the ground surface but nearer by the ceiling surface. Under monocular viewing conditions, the infants in both age groups reached preferentially for the toy that was specified as being nearer by the ground surface. This effect was significantly stronger than that observed under binocular viewing conditions. The findings indicate that the infants responded to the distance information provided by the ground surface to a greater extent than to information provided by the ceiling.
\end{abstract}

Keywords Infant vision - Perceptual development - Texture cues · Ground dominance effect · Pictorial depth cues · Depth perception $\cdot$ Distance perception

\footnotetext{
M. Kavšek ( $\square)$

Institute of Psychology, Department of Developmental

and Educational Psychology, University of Bonn,

Kaiser-Karl-Ring 9,

53111 Bonn, Germany

e-mail:kavsek@uni-bonn.de

C. E. Granrud

School of Psychological Sciences,

University of Northern Colorado,

Campus Box 94,

Greeley, CO 80639, USA

e-mail: Carl.Granrud@unco.edu
}

Gibson (1950) argued that the ground surface is uniquely important for spatial perception because we are terrestrial beings who depend on the ground surface for locomotion. Consistent with this view, research with adult participants has indicated that the ground surface plays a dominant role in the perception of spatial layout (e.g., Feria, Braunstein, \& Andersen, 2003; He, Wu, Ooi, Yarbrough, \& Wu, 2004; Meng \& Sedgwick, 2001, 2002; Sinai, Ooi, \& He, 1998; $\mathrm{Wu}, \mathrm{He}, \&$ Ooi, 2008). For example, Sinai et al. showed that the presence of discontinuities on a ground surface strongly affected distance perception and visually directed action.

If the ground surface is of special importance among the surfaces of our environment, it should be superior to ceiling surfaces and other planes in perceptual tasks. Indeed, several studies have provided evidence that visual search is made more efficient by a ground-like instead of a ceiling-like arrangement of the search array (McCarley \& He, 2000, 2001; see also Morita \& Kumada, 2003). Moreover, a ground surface facilitates 3-D size estimation (Champion \& Warren, 2010) and detection of change (Bian \& Andersen, 2010) more than ceilings and other surfaces do, and visual space is perceived as being less compressed when viewing a ground surface than when viewing a ceiling surface (Bian \& Andersen, 2011).

The ground surface also plays a dominant role in the perception of relative distances. Bian, Braunstein, and Andersen (2005) showed adult participants two rods surrounded by ground and ceiling surfaces. One of the rods covered more ground texture than ceiling texture, and the other covered more ceiling texture. As a result, the two surfaces provided contradictory information about the rods' distances. For example, in Fig. 1, which shows a display similar to those used by Bian et al. (2005), the ground surface specifies that the left rod/toy is near, and the ceiling specifies that it is far away. In contrast, the ground surface specifies the right rod/toy as being far away, and the ceiling specifies that it is near. Bian et al. (2005) found that the 


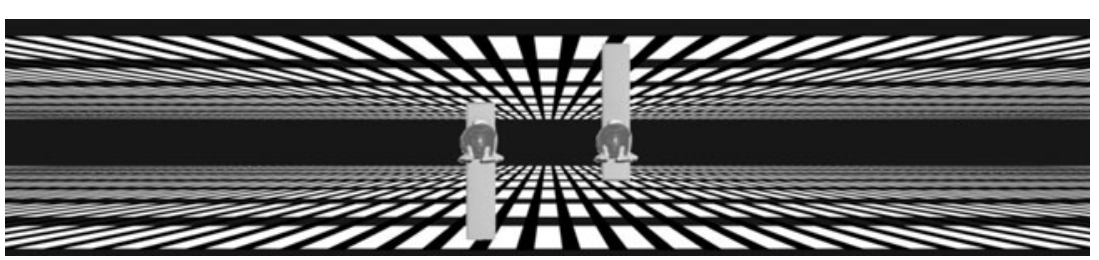

Fig. 1 Example of an experimental display. The ground and ceiling textures provide contradictory information about the relative distances of the two toys. The background texture was a rectangular picture, and the toys were rubber toys attached to rods. The rubber toy-rod combinations were held in place by magnets

found preferential reaching in 5- and 7-month-old infants. However, the reaching preference was stronger among the older than among the younger infants. In an additional condition, the ground texture was omitted from the display, making the differential relative heights of the toys the sole pictorial depth cue. Again, the 7-month-old infants reached preferentially for the apparently nearer toy, whereas the 5-month-old infants did not respond to the distance information specified by relative height in isolation. The 7-month-olds' reaching preference was significantly stronger in the texture-cues-plusrelative-height condition than in the relative-height-as-thesole-depth-cue condition. These findings suggest that infants 5 and 7 months of age respond to the distance information provided by texture cues and that relative height is a weaker source of information for distance. Hemker and Kavšek (2010) complemented this research by showing that infants are more sensitive to the linear perspective of the texture's surface contours than to the texture gradients of compression, perspective, and density.

The present study extended these earlier studies and examined whether infants display a ground dominance effect in perceiving objects' relative distances. Five- and 7 month-old infants were shown a display in which toys were affixed to rods that made contact with a textured ground and a textured ceiling (Fig. 1). To maximize the effectiveness of the distance information provided by surface texture, highly regular textures adapted from Hemker et al. (2010) were used. The rods' positions were manipulated so that the ground and ceiling provided contradictory information about the rods'/toys' relative distances. To minimize the impact of relative height, the toys were positioned at the same height.

The infants viewed the display under monocular (one eye patched) and binocular conditions, and their reaches toward the two toys were observed. Only reaches for the toys were analyzed; reaches to other parts of the display were not recorded. On the basis of previous findings that infants tend to reach toward the nearer of two objects, and that infants respond to depth illusions created by pictorial depth cues under monocular but not binocular viewing conditions (e.g., Yonas \& Granrud, 1985), we expected the infants to reach preferentially toward the toy that they perceived as being nearer in the monocular condition. The binocular condition 
served as a control for the possibility that infants might reach more often for one of the toys for a reason other than a perceived depth difference. If the infants' reaching behavior was determined by two-dimensional features within the display, no difference in reaching should be found between the viewing conditions, because these features were present in both conditions. If infants exhibited a stronger reaching preference for the apparently nearer toy (as specified by the ground surface) under monocular than under binocular conditions, this would provide evidence that they responded to the relative distance specified by texture on the ground in the monocular condition and to binocular information specifying that the toys were equidistant in the binocular condition.

\section{Method}

\section{Participants}

A group of 215 -month-old infants (10 females, 11 males; mean age $=153$ days, range $=148-160$ days $)$ and another of 27 7-month-old infants (13 females, 14 males; mean age = 214 days, range $=209-221$ days) participated in the experiment. In the 5-month-old group, 30 additional infants were not included in the final sample because of too few reaches $(n=7)$, fussiness $(n=18)$, or reaching for only one side in all trials of one or both (monocular and binocular) viewing conditions $(n=5)$. In the 7-month group, 24 additional infants were excluded because of too few reaches $(n=3)$, fussiness $(n=17)$, reaching for only one side in all trials of one or both viewing conditions $(n=3)$, or experimenter error $(n=1)$.

The participants were recruited by letter and follow-up telephone calls. The names of the infants were obtained from birth records provided by the municipal authorities of the city of Bonn, Germany. Data protection was guaranteed. The infants' parents were given toy animals. After the study, they were provided with information about the results of this research. All participants were treated in accord with the ethical guidelines of the American Psychological Association and the Society for Research in Child Development, and the study was approved by the ethics committee of the Institute of Psychology at the University of Bonn.

\section{Apparatus}

The display was a $119 \mathrm{~cm}$ wide $\times 27 \mathrm{~cm}$ high $\left(134.42^{\circ} \times\right.$ $56.74^{\circ}$ at a viewing distance of $25 \mathrm{~cm}$ ) rectangular picture. The display depicted two black-and-white, regularly spaced textured surfaces - a ground and a ceiling surface - that appeared to recede in depth (Fig. 1). The display was fixed on a white $170 \times 120 \mathrm{~cm}$ planar surface. Two black side panels $(185 \times 95 \mathrm{~cm})$, which were oriented at a $100^{\circ}$ angle from the experimental display, blocked the room and the experimenters from the infants' view. An orange-colored curtain $13.5 \mathrm{~cm}$ from the display could be lowered to cover it. Two cameras recorded the infants' reaching and looking behavior. One camera (model Hercules Classic Silver) was mounted above the display. It was directed downward and videotaped the infants' reaches. The other camera (model Logitech Webcam C905) was positioned in the middle of the display. This camera was used to observe the infant's looking and reaching behavior. The lens of the camera was black and was nearly indistinguishable from the black middle area of the display. The room was illuminated such that there were no reflections or shadows on the experimental display.

Stimuli

The textured surfaces consisted of white trapezoids on a black background. The ground surface was $11 \mathrm{~cm}$ high. The trapezoids in the lowest row of the ground surface were $1.8 \mathrm{~cm}$ away from the bottom edge of the display and $2.5 \mathrm{~cm}$ high. The upper row of trapezoids had a height of $0.4 \mathrm{~cm}$. The stripes of black background were $1 \mathrm{~cm}$ high at the bottom of the ground surface and $0.16 \mathrm{~cm}$ high at the top of the surface. The trapezoids' bottom contours were $3.2 \mathrm{~cm}$ wide at the bottom of the ground surface and $0.8 \mathrm{~cm}$ wide at the top. The black background stripes were $2 \mathrm{~cm}$ wide at the bottom and $0.4 \mathrm{~cm}$ wide at the top of the ground surface. The ceiling surface was constructed by rotating the ground surface by $180^{\circ}$. The distance between the ground and the ceiling surface was $5 \mathrm{~cm}$.

Two identical rubber toys attached to rods were affixed to the display (see Fig. 1), held in place by magnets. Each target (rubber toy-rod combination) was placed $7 \mathrm{~cm}$ in horizontal distance from the center of the display. Three pairs of targets were used: two orange penguins attached to orange rods, two green and yellow frogs attached to yellow rods, and two blue monkeys attached two blue rods. The rods' colors were similar to the rubber toys' colors in order to accentuate their coherence. When affixed to the display, the targets induced strong depth impressions. After the experiment, the parents were asked to describe the display. They consistently reported that they perceived the depth effect. None indicated that they perceived the rods as being slanted in depth. Furthermore, none reported that they perceived the upper target as being elevated above the ground. We additionally asked several adults whether they perceived the rods as slanted in depth or elevated above the ground. None answered either question in the affirmative. The rubber toys measured $4 \times 4 \times 4 \mathrm{~cm}$; the rods measured $3 \times$ $14.5 \mathrm{~cm}$. One rubber toy of each target pair was attached $10.25 \mathrm{~cm}$ above the lower edge of the rod, and the other 
rubber toy was attached $4.25 \mathrm{~cm}$ above the lower edge of the rod. When affixed to the display, the rubber toys were on the same horizontal axis. They were positioned in the black gap between the ground and the ceiling surfaces. One rod covered more texture of the ground than of the ceiling surface, and the other rod covered more texture of the ceiling than of the ground surface.

\section{Procedure}

Before testing began, the experimenters explained the procedure to the parents. The parents then read an information sheet and signed a consent form. The infants were given the opportunity to play with replicas of the experimental targets. The toys in these replicas were attached to the middle of the rods. The infants were given the targets in a random order for approximately $15 \mathrm{~s}$ each. The goal of this warm-up phase was to make the infants familiar with the unity of the rubber toys and the rods.

The parent was then seated on a chair with wheels that moved on a runner. The parent could easily roll the chair toward the display. The infant was seated on the parent's lap. Parents were instructed to hold their children around the waist and to keep them positioned in the middle of the two toys. This point was marked on the curtain, so that the infant's position could be adjusted. Other than monitoring the child's position, parents were asked not to interact with their children during the experiment. The study's hypotheses were explained after completion of the experimental session.

At the beginning of a trial, the infant was $45 \mathrm{~cm}$ from the display. If the infant did not orient to the display, a bell positioned behind the display was rung for approximately $5 \mathrm{~s}$ to attract the infant's attention. The parent was then instructed to roll toward the display. At the nearest point, the infant was approximately $25 \mathrm{~cm}$ from the display. The shoulder height of the infants was at the height of the rubber toys, such that both toys were equally easy to reach. When the infant had reached for one of the toys or when $30 \mathrm{~s}$ had elapsed without a reach occurring, the parent was instructed to roll away from the display. Then, the next trial was initiated.

At the beginning of the experiment and after every third trial, the display was covered by the curtain. One experimenter then changed the targets (penguins vs. monkeys vs. frogs) and their positions (lower rod on the left vs. lower rod on the right side). The orders of presentation of the targets were the same in both viewing conditions. The targets' initial position was randomly assigned to the participants.

The infants viewed the display in two blocks of trials: monocular and binocular. In the monocular condition, one eye was carefully occluded by a hypo-allergenic adhesive eye patch. It was randomly determined which eye was covered. Each viewing condition had a maximum of 18 trials. After the infant reached this maximum in the first condition, the infant took a 3- to 5-min break before the second condition started. Additional breaks were taken whenever the infant appeared to be tired or inattentive or if the infant did not reach for the rubber toys in several successive trials. If the infant was too fussy to continue, the experimental session was terminated. The first condition was stopped and was followed by the second condition after 18 trials or if it was necessary to take a break, provided that the infant had reached for one of the toys at least six times. Only infants who made at least six directed reaches in each condition were included in the final sample.

The order of the viewing conditions was counterbalanced across the sample. In the 5-month-old sample, 10 infants started with the monocular condition and 11 with the binocular condition. In the 7-month-old sample, 14 infants started with the monocular and 13 with the binocular condition.

Criteria for scoring infants' reaches Two experimenters observed the infant's looking and reaching behavior on a computer monitor attached to the two cameras that filmed the infant. The experimenters independently scored the direction of the infant's reaches by pressing buttons attached to a computer. The interrater agreement was very good (see, e.g., Landis \& Koch, 1977), $\kappa=.87$ (Cohen, 1960). If the two experimenters had scored a trial differently, they jointly coded the trial again from videotape.

Criteria for directed reaching Directed reaching for one of the two toys was scored if the infant looked at the toy before reaching for it. In the case of an infant reaching for both toys simultaneously, a directed reach was scored for the toy that was fixated. Reaching for the rods or any other contact with the display was scored as "without direction." Reaches "without direction" and trials in which no reach occurred were excluded from the data analysis. Only directed reaches were analyzed.

\section{Results}

Directed reaches were divided into reaches to the toy whose rod covered a greater proportion of the ground surface ("lower" target) and reaches to the toy whose rod covered a greater proportion of the ceiling surface ("upper" target). The percentage of reaches to the lower target was computed for both the monocular and binocular viewing conditions by dividing the number of reaches to the lower target by the total sum of reaches to the lower and upper targets. The percentages of reaches to the lower target were statistically analyzed. If the percentage of reaches to the lower target was significantly higher under monocular than under binocular viewing conditions, this would indicate that the infants responded to the pictorial depth cues in the experimental display. 
A preliminary data analysis found no effects of viewing condition order (monocular vs. binocular trials first) and initial target position (lower target on the left vs. the right). The data from these groups were therefore combined for subsequent analyses. Sex interacted significantly $(\alpha=.05)$ with age and viewing condition (monocular vs. binocular), $F(1,44)=11.16, p=.002, \eta_{\mathrm{P}}^{2}=.20$. The 5-month-old female infants exhibited a significantly higher percentage of reaches to the lower target in the monocular $(M=69.81$, $S D=11.56)$ than in the binocular $(M=49.31, S D=13.16)$ viewing condition, paired $t(9)=4.05$, two-tailed $p=.003$, effect size $d=1.28$. This effect was smaller and nonsignificant in the 7-month-old females (monocular, $M=61.65$, $S D=16.67$; binocular, $M=56.64, S D=12.55$ ). In the male sample, the 7-month-olds reached significantly more often for the lower target under monocular than under binocular conditions (monocular, $M=61.78, S D=14.90$; binocular, $M=41.95, S D=10.33)$, paired $t(13)=3.68$, two-tailed $p=$ $.003, d=0.98$. The 5-month-olds, however, exhibited no monocular-binocular difference (monocular, $M=57.14$, $S D=19.14$; binocular, $M=60.61, S D=8.61)$. These sex differences are difficult to explain and have not been observed in prior research on infant pictorial depth perception. They may be an artifact of having small samples when the participants are divided into groups of boys and girls. We therefore collapsed the data across both sexes.

Table 1 presents the mean numbers of directed reaches and the mean percentages of reaches to the lower target. Percentages of reaches to the lower target were analyzed in a $2 \times 2$ mixed ANOVA with age (5 vs. 7 months) as a between-subjects factor and viewing condition (monocular vs. binocular) as a within-subjects factor.

The analysis revealed a significant main effect for viewing condition, $F(1,46)=10.54, p=.002, \eta_{\mathrm{P}}{ }^{2}=.19$. The main effect of age did not reach statistical significance. Moreover, no significant interaction between viewing condition and age emerged. The main effect for viewing condition indicated a higher percentage of reaches to the lower target in the monocular (mean percentage $=62.36, S D=15.95$ ) than in the binocular $(M=51.74, S D=13.17)$ viewing condition.
A planned paired $t$ test was calculated for each age group to further investigate the effect of viewing condition on the percentages of reaches to the lower target. For the 5-monthold infants, the effect of viewing condition was significant in a one-tailed test, $t(20)=1.75, p=.048, d=0.38$ (see Table 1). For the 7-month-olds, the effect of viewing condition was highly significant in a two-tailed $t$ test, $t(26)=$ 2.91, $p=.007, d=0.56$ (see Table 1).

In sum, both age groups exhibited a stronger reaching preference for the lower target under monocular viewing than under binocular viewing. Planned analyses showed that this effect was slightly stronger in the older than in the younger participants. The overall ANOVA, however, did not reveal a substantial age difference: The effect of viewing condition was not modulated significantly by age.

In addition, planned one-sample $t$ tests were conducted, which compared the percentages of reaches to the lower target in each viewing condition to chance $(50 \%)$. The infants reached to the lower target significantly more often than $50 \%$ of the time in the monocular condition, $t(47)=$ $5.37, p \leq .001$ (two-tailed), $d=0.78$, but not in the binocular condition, $t(47)=0.91, p=.37$. Similarly, analyses for each age group separately showed that both the 5-month-olds, $t(20)=3.58, p=.002, d=0.78$, and the 7-month-olds, $t(26)=3.94, p=.001, d=0.76$, reached significantly more than $50 \%$ of the time for the lower target in the monocular viewing condition. In the binocular condition, the percentage of reaches to the lower target was marginally greater than $50 \%$ for the 5 -month-olds, $t(20)=1.97, p=.063$, and did not differ from chance for the 7-month-olds, $t(26)=-0.38, p=.71$.

Participant loss was high, which is typical in preferential reaching studies (e.g., Corrow, Granrud, Mathison, \& Yonas, 2011, 2012; Yonas \& Granrud, 2006). This was probably due, in part, to our strict inclusion criteria. For example, all reaches in which the infants did not look at the toy for which they were reaching were excluded from the data analysis (see also Hemker et al., 2010). However, Slaughter and Suddendorf (2007), in their review on participant loss in infant vision studies, found no correlation between exclusion rates and experimental outcomes. Furthermore, we

Table 1 Mean number of directed reaches and mean percentage of reaches to the lower target in each viewing condition

\begin{tabular}{|c|c|c|c|c|c|}
\hline \multirow[t]{2}{*}{ Age } & \multirow[t]{2}{*}{ Viewing Condition } & \multicolumn{2}{|c|}{ Number of Directed Reaches } & \multicolumn{2}{|c|}{$\begin{array}{l}\text { Percentage of Reaches to the Lower } \\
\text { Target }\end{array}$} \\
\hline & & $M$ & $S D$ & $M$ & $S D$ \\
\hline \multirow[t]{2}{*}{5 months } & Monocular & 11.0 & 3.44 & 63.18 & 16.89 \\
\hline & Binocular & 13.05 & 3.28 & 55.23 & 12.18 \\
\hline \multirow[t]{2}{*}{7 months } & Monocular & 13.0 & 3.69 & 61.72 & 15.47 \\
\hline & Binocular & 13.15 & 3.23 & 49.02 & 13.49 \\
\hline
\end{tabular}


Table 2 Mean percentage of reaches to the lower target in each viewing condition by infants excluded from the sample

\begin{tabular}{llll}
\hline Age & Viewing Condition & \multicolumn{2}{l}{$\begin{array}{l}\text { Percentage of Reaches to the } \\
\text { Lower Target }\end{array}$} \\
\cline { 3 - 4 } & & $M$ & $S D$ \\
\hline 5 months $(n=11)$ & Monocular & 59.97 & 34.87 \\
& Binocular & 51.79 & 11.50 \\
7 months $(n=10)$ & Monocular & 66.20 & 23.35 \\
& Binocular & 58.78 & 16.27 \\
\hline
\end{tabular}

conducted an analysis of the participants who produced data in both the monocular and binocular viewing conditions, but who were excluded for not satisfying the selection criteria, so as to examine whether their reaching behavior was consistent with the general findings. Table 2 summarizes the mean percentages of reaches to the lower target in each viewing condition for these participants. The excluded 5- and 7-month-old infants reached more often for the lower target in the monocular than in the binocular condition. They therefore behaved similarly to the infants who were included in the sample, and their exclusion did not affect the results of the study.

\section{Discussion}

Under monocular viewing conditions, the 5- and 7-monthold infants reached more often for a toy that was specified as being nearer by the ground surface than for a toy that was specified as being nearer by the ceiling surface. These infants therefore exhibited a ground dominance effect: They attended to the distance information provided by the ground surface more than to information provided by the ceiling. The results indicate that the ground plays a dominant role in distance perception and visually guided reaching at a very early age, before infants can crawl and walk. The finding of a weaker reaching preference for the lower target in the binocular condition indicates that the infants' monocular reaching preference cannot be explained as a response to two-dimensional display features such as the toys' positions relative to the tops of the rods. Moreover, the weaker reaching preference under binocular viewing is inconsistent with the assumption that the ground dominance effect is a general bias to attend to the lower part of the visual field. If so, the infants would have shown similar reaching preferences for the lower target under monocular and binocular viewing conditions. The results, therefore, indicate that the infants' monocular reaching behavior was based on the information provided by surface texture that specified a difference in the distances of the two toys.
The overall data analyses indicated that the 5- and the 7month-old infants displayed the ground dominance effect and sensitivity to the distance information provided by surface texture. Although no statistically significant age differences were found, the effect was somewhat weaker in the 5-month-old than in the 7-month-old infants. This finding is consistent with the preferential reaching results found by Hemker et al. (2010), who demonstrated sensitivity to the depth information provided by a textured ground surface in 5- and 7-month-old infants, as well as a stronger response to texture cues in the older age group.

An alternative explanation for this study's results might be that the infants responded to the differential relative heights of the targets and not to their perceived relative distances. This interpretation, however, is unlikely for several reasons. First, Bian, Braunstein, and Andersen (2005, 2006) did not find an effect of differences in the rods' heights on adults' distance judgments. Consistent with this observation, several studies have found that relative height is a weak depth cue for adults (e.g., Rock, Shallo, \& Schwartz, 1978) as well as for children (e.g., McGurk \& Jahoda, 1974). Furthermore, we observed reaching for toys positioned at the same height, and the height differences between the rods was smaller than that in the Hemker et al. (2010) study. That study employed a display very similar to that used in the present study, and Hemker et al. established a reliable effect of texture cues in infants 5 and 7 months of age: Relative height in isolation had either no (5-month-olds) or a significantly smaller (7-month-olds) effect on infants' reaching (but see Arterberry, 2008). Nevertheless, further research will be needed to further examine the impact of relative height information.

The findings from several studies have indicated that the infant visual system uses heuristic assumptions to disambiguate two-dimensional retinal image cues to extract information about three-dimensional object shape and spatial layout. For example, Imura, Tomonaga, Yamaguchi, and Yagi (2008) and Shirai, Kanazawa, and Yamaguchi (2005) tested infants' sensitivity to shading and found that 4- and 5month-old infants behaved as if they made use of the assumption that objects are usually lit from above. More recently, Corrow et al. (2011) established that 6-month-old infants use the assumption that faces are convex, and Corrow et al. (2012) found that 5- and 7-month-olds employ an assumption that objects in general are convex in perceiving three-dimensional object shape. The present findings suggest that the ground dominance effect, the assumption that objects' distances are primarily specified by their positions on a ground surface, is a basic constraint that is also applied by the infants' visual system. This is consistent with the assumption that our visual system has adapted to the depth information provided by ground texture because our locomotion is usually confined to the ground surface (e.g., Gibson, 1950; 
Nakayama, He, \& Shimojo, 1995). As a consequence, the ground dominance effect may be inherent in our visual system from birth onward. Alternatively, our visual system may be tuned to quickly learn to extract depth information from ground texture during the first months of life.

Our experiment replicated and extended numerous preferential reaching studies that had observed that 5- and 7month-old infants are sensitive to pictorial depth cues, such as surface texture (e.g., Hemker et al., 2010; Yonas et al., 1986; for a review, see Kavšek, Granrud, \& Yonas, 2009). It suggests that the ground surface is superior to the ceiling surface in the perception of distance and the planning of directed reaching from 5 months onward.

It remains unknown, however, whether responsiveness to depth from texture and the ground dominance effect are present earlier in life. Studies using looking methods have found responsiveness to several pictorial depth cues in infants as young as 3 months (e.g., Bertin \& Bhatt, 2006; Tsuruhara et al., 2010; for a review, see Kavšek, Yonas, \& Granrud, 2012). Future research will be needed to determine whether the ground dominance effect can be found in infants 3 months of age or younger.

Author note The authors thank Gesa Schlachter and Mirjam Ernst for research assistance. Thanks are especially extended to the infants and parents who participated in the study.

\section{References}

Arterberry, M. (2008). Infants' sensitivity to the depth cue of height-inthe-picture-plane. Infancy, 13, 544-555.

Arterberry, M., Yonas, A., \& Bensen, A. S. (1989). Self-produced locomotion and the development of responsiveness to linear perspective and texture gradients. Developmental Psychology, 25, 976-982.

Bertin, E., \& Bhatt, R. S. (2006). Three-month-olds' sensitivity to orientation cues in the three-dimensional depth plane. Journal of Experimental Child Psychology, 93, 45-62.

Bian, Z., \& Andersen, G. J. (2008). Aging and the perceptual organization of 3-D scenes. Psychology and Aging, 23, 342-352.

Bian, Z., \& Andersen, G. J. (2010). The advantage of a ground surface in the representation of visual scenes. Journal of Vision, 10(8):16, 1-9. doi:10.1167/10.8.16

Bian, Z., \& Andersen, G. J. (2011). Environmental surfaces and the compression of perceived visual space. Journal of Vision, 11(7):4, 1-14. doi:10.1167/11.7.4

Bian, Z., Braunstein, M. L., \& Andersen, G. J. (2005). The ground dominance effect in the perception of 3-D layout. Perception \& Psychophysics, 67, 802-815. doi:10.3758/BF03193534

Bian, Z., Braunstein, M. L., \& Andersen, G. J. (2006). The ground dominance effect in the perception of relative distance in 3-D scenes is mainly due to characteristics of the ground surface. Perception \& Psychophysics, 68, 1297-1309. doi:10.3758/ BF03193729

Champion, R. A., \& Warren, P. A. (2010). Ground-plane influences on size estimation in early visual processing. Vision Research, 50, $1510-1518$.
Cohen, J. (1960). A coefficient of agreement for nominal scales. Educational and Psychological Measurement, 20, 37-46.

Corrow, S., Granrud, C. E., Mathison, J., \& Yonas, A. (2011). Sixmonth-old infants perceive the hollow-face illusion. Perception, 40, 1376-1383.

Corrow, S., Granrud, C. E., Mathison, J., \& Yonas, A. (2012). Infants and adults use line junction information to perceive 3D shape. Journal of Vision, 12(1):8, 1-7. doi:10.1167/12.1.8

Feria, C. S., Braunstein, M. L., \& Andersen, G. L. (2003). Judging distance across texture discontinuities. Perception, 32, 14231440.

Gibson, J. J. (1950). The perception of the visual world. Cambridge, MA: Riverside Press.

He, Z. J., Wu, B., Ooi, T. L., Yarbrough, G., \& Wu, J. (2004). Judging egocentric distance on the ground: Occlusion and surface integration. Perception, 33, 789-806.

Hemker, L., Granrud, C. E., Yonas, A., \& Kavšek, M. (2010). Infant perception of surface texture and relative height as distance information: A preferential-reaching study. Infancy, 15, 6-27.

Hemker, L., \& Kavšek, M. (2010). The relative contribution of relative height, linear perspective, and texture gradients to pictorial depth perception in 7-month-old infants. Perception, 39, 1476-1490.

Imura, T., Tomonaga, M., Yamaguchi, M. K., \& Yagi, A. (2008). Asymmetry in the detection of shapes from shading in infants. Japanese Psychological Research, 50, 128-136.

Kavšek, M., Granrud, C. E., \& Yonas, A. (2009). Infants' responsiveness to pictorial depth cues in preferential-reaching studies: A meta-analysis. Infant Behavior and Development, 32, 245-253.

Kavšek, M., Yonas, A., \& Granrud, C. E. (2012). Infants' sensitivity to pictorial depth cues: A review and meta-analysis of looking studies. Infant Behavior and Development, 35, 109-128.

Landis, J. R., \& Koch, G. G. (1977). The measurement of observer agreement for categorical data. Biometrics, 33, 159-174.

McCarley, J. S., \& He, Z. J. (2000). Asymmetry in 3-D perceptual organization: Ground-like surface superior to ceiling-like surface. Perception \& Psychophysics, 62, 540-549.

McCarley, J. S., \& He, Z. J. (2001). Sequential priming of 3-D perceptual organization. Perception \& Psychophysics, 63, 195208.

McGurk, H., \& Jahoda, G. (1974). The development of pictorial depth perception: The role of figural elevation. British Journal of Psychology, 65, 367-376.

Meng, J. C., \& Sedgwick, H. A. (2001). Distance perception meditated through nested contact relations among surfaces. Perception \& Psychophysics, 63, 1-15.

Meng, J. C., \& Sedgwick, H. A. (2002). Distance perception across spatial discontinuities. Perception \& Psychophysics, 64, 1-14.

Morita, H., \& Kumada, T. (2003). Effects of pictorially-defined surfaces on visual search. Vision Research, 43, 1869-1877.

Nakayama, K., He, Z. J., \& Shimojo, S. (1995). Visual surface representation: A critical link between lower-level and higher level vision. In D. N. Osherson \& S. M. Kosslyn (Eds.), An invitation to cognitive science: Vol. 2. Visual cognition (pp. 1-70). Cambridge, MA: MIT Press.

Rock, I., Shallo, J., \& Schwartz, F. (1978). Pictorial depth and related constancy effects as a function of recognition. Perception, 7, 3-19.

Shirai, N., Kanazawa, S., \& Yamaguchi, M. K. (2005). Young infants' sensitivity to shading stimuli with radial motion. Japanese Psychological Research, 47, 286-291.

Sinai, M. J., Ooi, T. L., \& He, Z. J. (1998). Terrain influences the accurate judgement of distance. Nature, 395, 497-500.

Slaughter, V., \& Suddendorf, T. (2007). Participant loss due to "fussiness" in infant visual paradigms: A review of the last 20 years. Infant Behavior and Development, 30, 505-514.

Tsuruhara, A., Sawada, T., Kanazawa, S., Yamaguchi, M. K., Corrow, S., \& Yonas, A. (2010). The development of the ability of infants 
to utilize static cues to create and access representations of object shape. Journal of Vision, 10(12):2, 1-11. doi:10.1167/10.12.2

Wu, J., He, Z. J., \& Ooi, T. L. (2008). Perceived relative distance on the ground affected by the selection of depth information. Perception \& Psychophysics, 70, 707-713. doi:10.3758/PP.70.4.707

Yonas, A., Elieff, C. A., \& Arterberry, M. E. (2002). Emergence of sensitivity to pictorial depth cues: Charting development in individual infants. Infant Behavior and Development, 25, 495514.
Yonas, A., \& Granrud, C. E. (1985). Reaching as a measure of infants' spatial perception. In G. Gottlieb \& N. A. Krasnegor (Eds.), Measurement of audition and vision in the first year of postnatal life: A methodological overview (pp. 301-322). Norwood, NJ: Ablex.

Yonas, A., \& Granrud, C. E. (2006). Infants' perception of depth from cast shadows. Perception \& Psychophysics, 68, 154-160.

Yonas, A., Granrud, C. E., Arterberry, M. E., \& Hanson, B. L. (1986). Infants' distance perception from linear perspective and texture gradients. Infant Behavior and Development, 9, 247-256. 